\title{
$\alpha v$ Integrin expression by DCs is required for Th17 cell differentiation and development of experimental autoimmune encephalomyelitis in mice
}

Mridu Acharya 1 Subhankar Mukhopadhyay, 1,2 Helena Païdassi, 1,3 Tahseen Jamil,1 Camille Chow, Stephan Kissler, ${ }^{4}$ Lynda M. Stuart, ${ }^{1}$ Richard O. Hynes, ${ }^{4,5}$ and Adam Lacy-Hulbert ${ }^{1}$

\begin{abstract}
${ }^{1}$ Program of Developmental Immunology, Department of Pediatrics, Massachusetts General Hospital and Harvard Medical School, Boston, Massachusetts, USA. ${ }^{2}$ Sir William Dunn School of Pathology, University of Oxford, Oxford, United Kingdom.

${ }^{3}$ Queen's Medical Research Institute and MRC Centre for Inflammation Research, University of Edinburgh, Edinburgh, United Kingdom. ${ }^{4}$ Koch Institute for Integrated Cancer Research, Massachusetts Institute of Technology, Cambridge, Massachusetts, USA. ${ }^{5}$ Howard Hughes Medical Institute, Chevy Chase, Maryland, USA.
\end{abstract}

\begin{abstract}
Th17 cells are a distinct lineage of $\mathrm{T}$ helper cells that protect the body from bacterial and fungal infection. However, Th17 cells also contribute to inflammatory and autoimmune disorders such as multiple sclerosis. Th17 cell generation requires exposure of naive T cells to the cytokine TGF- $\beta$ in combination with proinflammatory cytokines. Here we show that differentiation of Th17 cells is also critically dependent on $\alpha \mathrm{v}$ integrins. In mice, lack of integrin $\alpha v$ in the immune system resulted in loss of Th17 cells in the intestine and lymphoid tissues. It also led to protection from experimental autoimmune encephalomyelitis (EAE). Further analysis indicated that $\alpha v$ integrins on DCs activated latent TGF- $\beta$ during T cell stimulation and thereby promoted differentiation of Th17 cells. Furthermore, pharmacologic inhibition of $\alpha v$ integrins using cyclic RGD peptides blocked TGF- $\beta$ activation and Th17 cell generation in vitro and protected mice from EAE. These data demonstrate that activation of TGF- $\beta$ by $\alpha$-expressing myeloid cells may be a critical step in the generation of Th17 cells and suggest that $\alpha v$ integrins could be therapeutic targets in autoimmune disease.
\end{abstract}

\section{Introduction}

Th17 cells are a recently described subset of $\mathrm{T}$ helper cells distinct from Th1 and Th2 cells (1-4). They were initially characterized by expression of IL-17A and IL-17F, but also express IL-21 and IL-22 in addition to other cytokines, and are defined by expression of the transcription factor ROR- $\gamma \mathrm{T}$ (5). Th17 cells are an important component of adaptive immune responses to extracellular bacteria and fungi at mucosal surfaces and are most prevalent in the intestinal lamina propria (LP) (3), where they are generated in response to colonization by microbes such as segmented filamentous bacteria $(6,7)$. In the intestine, Th17 cells protect against infection and also mediate intestinal homeostasis though expression of IL-17A and IL-22 $(8,9)$. In contrast, Th17 cells also act as pathogenic effectors in several mouse models of autoimmunity, most notably in experimental autoimmune encephalomyelitis (EAE), the mouse model of multiple sclerosis (10). Recent cellular and genetic association studies have also linked Th17 cells to a wide range of human chronic inflammatory and autoimmune disorders, including multiple sclerosis, rheumatoid arthritis, and Crohn disease $(4,11,12)$. However, progress in understanding the role of Th17 cells in human disease is complicated due to their apparent plasticity (13) and overlapping patterns of cytokine expression between Th17 and other immune cell populations, and additional tools to selectively target $\mathrm{Th} 17$-responses are needed.

Th17 differentiation is critically dependent on TGF- $\beta$, in combination with IL-6 or IL-21 (14-16). TGF- $\beta$ also promotes differentiation of adaptive Tregs (aTregs), and Th17 cells and Tregs

Conflict of interest: The authors have declared that no conflict of interest exists. Citation for this article: $J$ Clin Invest. 2010;120(12):4445-4452. doi:10.1172/JCI43796. share a common precursor that expresses both ROR- $\gamma \mathrm{T}$ and the Treg-specific transcription factor FoxP3 (17). TGF- $\beta$ is synthesized as an inactive latent precursor that requires cleavage and/or dissociation from the latency-associated peptide (LAP) to engage the TGF- $\beta$ receptor and signal. $\alpha$ Integrins are important physiological regulators of TGF- $\beta$ activation, and deletion of $\alpha \mathrm{v}$ integrins or disruption of the $\alpha v$-binding site in TGF- $\beta$ causes failure of effective TGF- $\beta$ signaling in vivo (18-20). We have previously shown that deletion of $\alpha v$ from myeloid cells leads to loss of intestinal Tregs and development of spontaneous colitis, which we attribute to failure of TGF- $\beta$ activation by DCs and loss of TGF- $\beta$ signaling to $T$ cells (21). Considering this observation and the common requirement for TGF- $\beta$ in early commitment of both Tregs and Th17 cells, we set out to determine whether Th17 cell generation may also be regulated by $\alpha v$ integrins.

\section{Results}

$\alpha v$-Deficient mice lack intestinal Th17 cells due to loss of $\alpha v$ from myeloid cells. We first analyzed T cells isolated from the LP of av-tie2 mice, which lack $\alpha v$ integrins in all hematopoietic cells (21). The proportion of Th17 cells (determined either by high expression of the transcription factor ROR- $\gamma \mathrm{T}$ or by production of IL-17) was significantly reduced in the intestines of $\alpha v$-tie 2 mice, consistent with a role for $\alpha v$ integrins in Th17 cell development. Indeed, deletion of $\alpha v$ integrins had a more significant effect on Th17 cells (7-fold reduction) than on FoxP3 ${ }^{+}$Tregs (3-fold reduction; Figure 1A). Similar reductions in the proportions of Th17 cells were seen in lymphoid tissues, and in all cases the absolute numbers of Th17 cells were also reduced (data not shown). In contrast, IFN- $\gamma$-producing Th1 
A
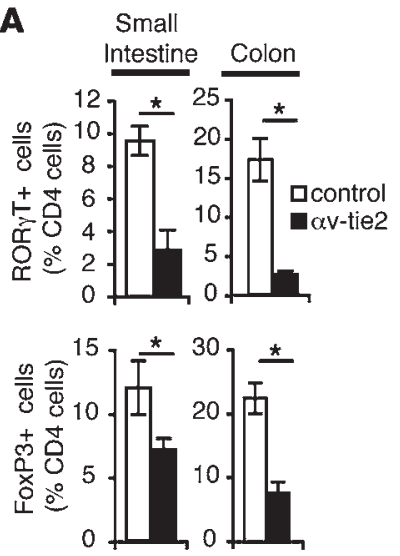

D

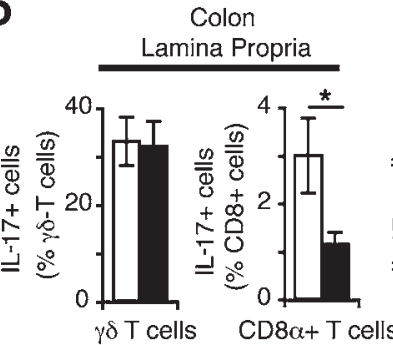

B

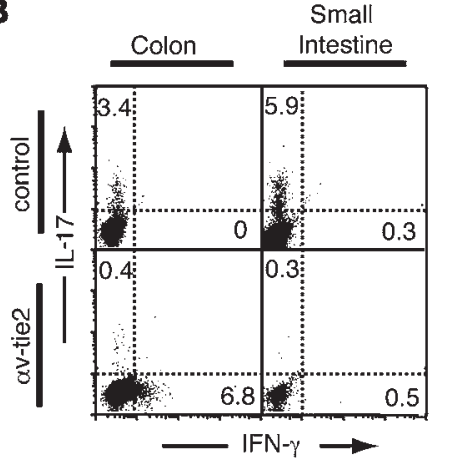

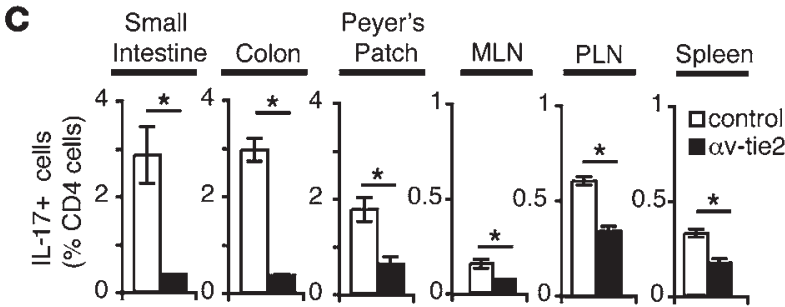

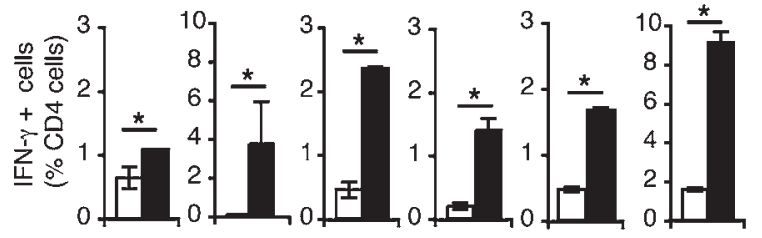

Peritoneal cavity
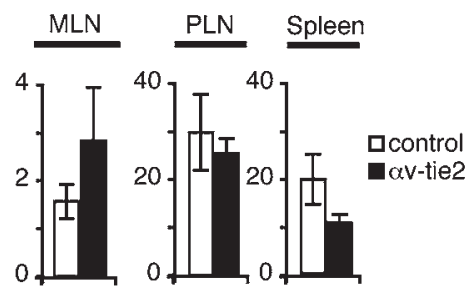

E

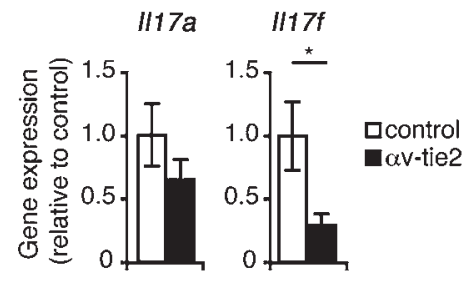

\section{Figure 1}

Lack of intestinal Th17 cells in $\alpha$-tie2 mice. (A) Proportion of ROR- $\gamma \mathrm{T}^{+}$and FoxP3 ${ }^{+}$CD4 $4^{+}$cells in LP of control and $\alpha \mathrm{V}$-tie2 mice. (B and C) Proportion of IL-17-producing (Th17 cells) and IFN- $\gamma$-producing (Th1 cells) CD4+ $4^{+}$cells isolated from the LP of the small intestine and colon, Peyer's patch, MLN, peripheral LN (PLN), and spleen of control and av-tie2 mice. (B) Representative FACS data from one experiment. The plots are gated on $\mathrm{CD}^{+}$cells, and the numbers represent the percentage of CD4+ cells that stained for IL-17 or IFN- $\gamma$. (C) Mean \pm SEM from at least 3 mice/group. (D) Proportion of IL-17-producing $\gamma \delta$ and CD $8 \alpha^{+}$T cells isolated from the LP of the colon, and IL-17-producing $\gamma \delta$ T cells from indicated tissues, of control and $\alpha$-tie2 mice. (E) Expression of $/ / 17 a$ and I/17f measured by QRT-PCR in RNA isolated from proximal colon of control and $\alpha v$-tie2 mice. All graphs show mean \pm SEM from at least 3 mice/group. ${ }^{*} P<0.05$.

cells were expanded in the intestine and lymphoid organs (Figure 1, $\mathrm{B}$ and C). Furthermore, other IL-17-producing lymphocyte populations appeared to be largely unaffected by deletion of $\alpha \mathrm{v}$. In particular, $\gamma \delta$ T cells, a major source of IL-17 in vivo, were present in similar numbers in control and $\alpha v$-tie 2 mice (data not shown) and showed equivalent levels of IL-17 production (Figure 1D). Consequently, expression of Il17a was not significantly decreased in the intestine of $\alpha v$-tie2 mice, although $I l 17 f$ was reduced 3-fold, suggesting that Th17 cells contribute significantly to the production of this cytokine in the intestine (Figure 1E). We therefore concluded that deletion of $\alpha v$ integrins from immune cells led to loss of Th17 cells but did not affect other IL-17-producing cells.

Loss of Th17 cells was not due to intrinsic defects in $\alpha \mathrm{v}$-deficient $\mathrm{T}$ cells, as $\mathrm{CD} 4^{+} \mathrm{T}$ cells from $\alpha v$-tie 2 mice differentiated normally into Th17 cells when adoptively transferred into $\alpha v$-expressing SCID mice (Figure 2A). Instead, $\alpha v$ expression by innate immune cells was required for differentiation of naive cells to Th17 cells, as $\mathrm{T}$ cells from wild-type mice did not become Th17 cells when transferred to $\alpha v$-tie2/ SCID recipients (Figure 2B). To determine whether this was due to deletion of $\alpha v$ from myeloid cells, we analyzed Th17 cells in $\alpha v$-LysM mice, in which $\alpha v$ is deleted specifically from macrophages, DCs, and neutrophils. Th17 cell numbers were significantly decreased in $\alpha v$-LysM mice (Figure 2C), similar to our previous observations that the generation of intestinal Tregs required $\alpha v$ expression by DCs (21). In addition, the proportion of IFN- $\gamma$-producing cells was not markedly increased in these mice, suggesting that the loss of Th17 cells was not due to expansion of Th1 cells, as has been proposed in other studies (22). Together, these data show that Th17 cells were reduced in $\alpha v$-deficient mice and that $\alpha v$ expression by myeloid cells was required to establish the intestinal Th17 population.

$\alpha v$-Deficient mice do not develop pathological Th17 cells and are protected from $E A E$. In contrast to their protective role in mucosal immunity $(8,9)$, Th17 cells are implicated in immune-mediated diseases and are critical for development of EAE (10). To establish whether $\alpha v$ integrins were involved in generation of pathological Th17 cells, we tested whether $\alpha v$-knockout mice were protected from EAE. Induction of EAE by immunization with myelin oligodendrocyte glycoprotein-derived (MOG-derived) peptide in control mice resulted in generation of Th17 cells in the LNs and spleen (Figure 3A) with subsequent infiltration into the brain and associated paralysis (Figure 3, $\mathrm{B}-\mathrm{E})$. In contrast, immunization of $\alpha \mathrm{v}$-tie 2 mice induced neither significant numbers of Th17 cells nor clinical signs of EAE (Figure 3). Thus, we concluded that $\alpha v$-tie2 mice fail to generate pathological Th17 cells, resulting in complete protection from EAE.

Building on these observations, we next assessed whether protection from EAE was due to loss of $\alpha v$ from $T$ cells or from myeloid cells. Mice in which $\alpha v$ was deleted from T cells ( $\alpha v-C D 4$ mice) developed brain-infiltrating Th17 cells and paralysis to the same degree as littermate controls, demonstrating that $\alpha v$ was not required on $\mathrm{T}$ cells for pathological Th17 development (Figure 3, F and G). 
A

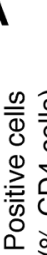

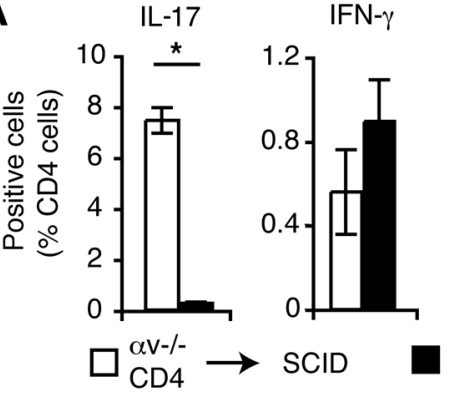

FoxP3

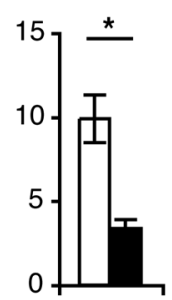

av-tie2; SCID

B
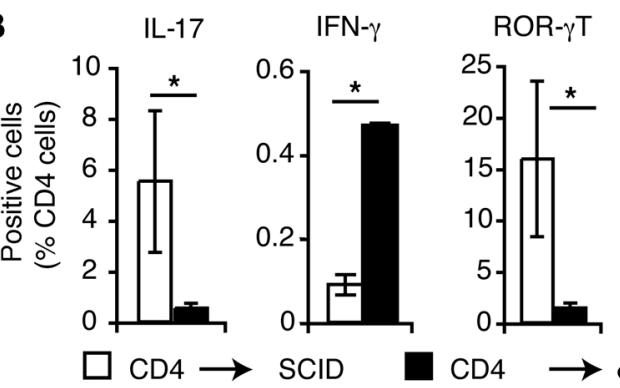

FoxP3

C
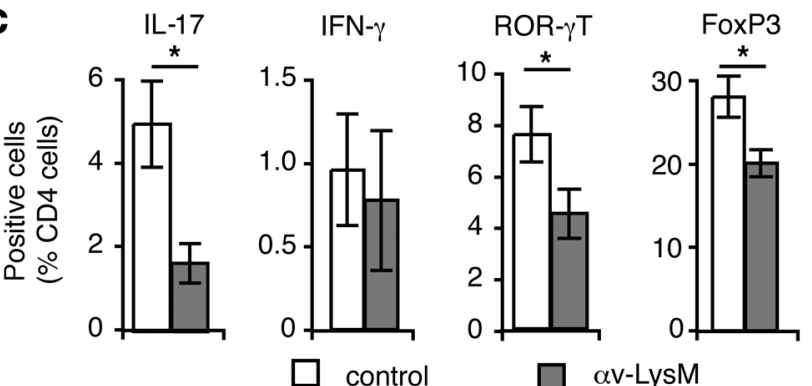

In contrast, $\alpha v$-LysM mice were protected from disease, with delayed development of paralysis (18 days post-immunization in $\alpha v$-LysM mice compared with 12 days in controls) and reduced disease severity when compared with controls (mean disease score at sacrifice of $1.4 \pm 0.8$ vs. $2.6 \pm 0.3$ in $\alpha$ v-LysM and controls, respectively; Figure 3, $\mathrm{H}$ and I). Furthermore, Th17 cells were reduced 3-fold in the brain of av-LysM mice with EAE, confirming that $\alpha v$ expression by myeloid cells was essential for generation of pathological Th17 cells. The protection was less than that seen with av-tie 2 mice, probably due to incomplete deletion of $\alpha \mathrm{v}$ from myeloid cells in $\alpha v$-LysM mice (21). These results demonstrate that generation of pathological Th17 cells and susceptibility to Th17-mediated disease required av expression by myeloid cells but not $\mathrm{T}$ cells.

$D C \alpha v$ integrins activate latent TGF- $\beta$ for generation of Th17 cells. We next set out to determine the mechanism by which myeloid cell $\alpha v$ integrins promoted Th17 cell generation. As we have reported previously, DCs appear to develop normally in $\alpha v$-tie2 mice and migrate to tissues and LNs (ref. 21 and Supplemental Figure 1; supplemental material available online with this article; doi:10.1172/JCI43796DS1). Furthermore, $\alpha v$-deficient DCs produced normal levels of the Th17-promoting proinflammatory cytokines IL- 6 and IL-23 when stimulated in vitro, and these cytokines were expressed in the intestine of $\alpha \mathrm{v}$-tie2 mice at similar or higher levels than those in control mice (Supplemental Figures 1 and 2). It was therefore unlikely that loss of Th17 cells

\section{Figure 2}

$\alpha$ V Expression by myeloid cells is required for Th17 cell differentiation. (A and B) Tregs and Th17 and Th1 cells in the colonic LP of SCID control and $\alpha$ V-tie2/ SCID mice 6 weeks following adoptive transfer with splenic CD4+ $T$ cells from $\alpha v$-tie2 (A) or wild-type control (B) mice. Data are from 4 littermate recipients/group. (C) Th17 and Th1 cells and Tregs in the colonic LP of control and $\alpha$ V-LysM mice (12 weeks of age). Data are from 4 littermates/group. In all cases, similar differences were seen in at least 3 independent experiments. ${ }^{*} P<0.05$, Student's $t$ test.

was due to defects in antigen presentation or proinflammatory cytokine production by DCs. Instead, the defects in generation of both Th17 cells and Tregs in av-tie2 mice implicated defects in TGF- $\beta$ signaling. An important and nonredundant function of $\alpha v$ integrins in vivo is to bind latent TGF- $\beta$ and catalyze conversion to an active form (18-20). Our previous work, and that of others, has suggested that DCs express $\alpha v \beta 8$, which allows them to bind and activate TGF- $\beta$, which is then available for paracrine signaling to cognate $T$ cells $(21,23)$. $\alpha$ v Integrin-mediated binding to TGF- $\beta$ occurs through an Arg-Gly-Asp (RGD) tripeptide in the LAP (24). We found that DCs were able to bind both LAP and the RGD-containing integrin ligand fibronectin, and this was dependent on $\alpha \mathrm{v}$ expression (Figure 4A). To test whether DCs could activate TGF- $\beta$ for signaling to T cells and promotion of Th17 differentiation, naive $\mathrm{CD} 4^{+} \mathrm{T}$ cells were co-cultured with DCs in the presence of IL- 6 with active or latent TGF- $\beta$. DCs cultured with T cells alone or in the presence of IL- 6 alone did not generate significant numbers of Th17 cells, whereas addition of active TGF- $\beta$ stimulated generation of Th17 cells by both wildtype and $\alpha v$-deficient DCs to an equivalent degree, as assessed by IL-17 production and expression of rorc (Figure 4, B-D). In contrast, increasing concentrations of latent TGF- $\beta$ stimulated significantly higher proportions of Th17 cells in cultures of wild-type DCs than those with av-deficient DCs (Figure 4, B-D). Generation of Th17 cells was independent of $\mathrm{T}$ cell expression of $\alpha v$, as $\alpha v$-deficient $T$ cells became Th17 cells in response to both active and latent TGF- $\beta$ when co-cultured with wild-type DCs (data not shown). We next tested whether there needed to be a cognate interaction between the $\alpha$ v-expressing DC and the $T$ cell, or whether bystander DCs that could not present antigen could nonetheless activate latent TGF- $\beta$ and rescue the defect in $\alpha v$ knockouts. Using OT-2 TCR transgenic T cells, which respond to ovalbumin-derived peptide in the context of I- $\mathrm{A}^{\mathrm{b}}$, we showed that DCs of C57BL/ 6 background (which express I- $\mathrm{A}^{\mathrm{b}}$ ) could generate Th17 cells in the presence of latent TGF- $\beta$, and that this required $\alpha \mathrm{v}$ expression by the DCs. This could not be rescued by addition of wild-type DCs of BALB/c background, which expressed $\alpha v$ but could not present antigen to OT-2 T cells (Figure 4E). Together these data indicate that $\alpha \mathrm{v}$ expression by DCs is required for interacting $\mathrm{T}$ cells to respond to latent, but not active, TGF- $\beta$. We conclude that, during antigen presentation, DC $\alpha$ v activates latent TGF- $\beta$, which can then signal to the interacting $\mathrm{T}$ cells and promote Th17 differentiation.

RGD peptide mimetics inbibit Th17 differentiation and protect from EAE. Small molecule mimetics of RGD have been used clinically to inhibit integrin-mediated adhesion and signaling in tumors, principally glioblastoma (25). Using our in vitro co-culture system and a cyclic RGD (cRGD) peptide selective for $\alpha \mathrm{v}$ integrins (26), we tested whether RGD mimetics might also block the ability of $\alpha \mathrm{v}$ expressed by DCs to bind and activate TGF- $\beta$, and hence 
A

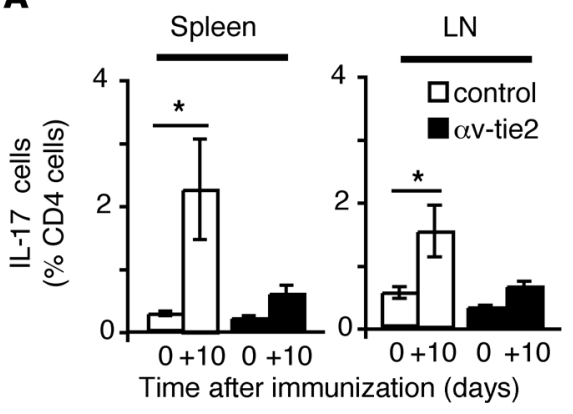

B

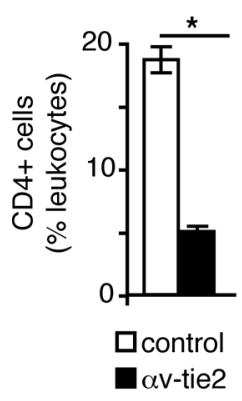

C

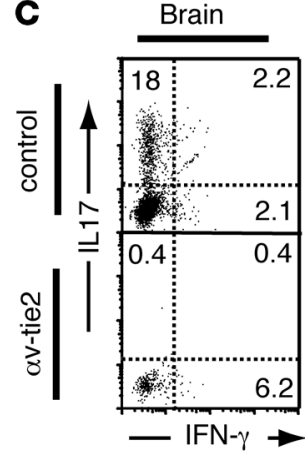

D

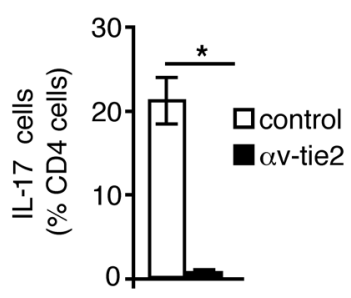

$\mathbf{F}$
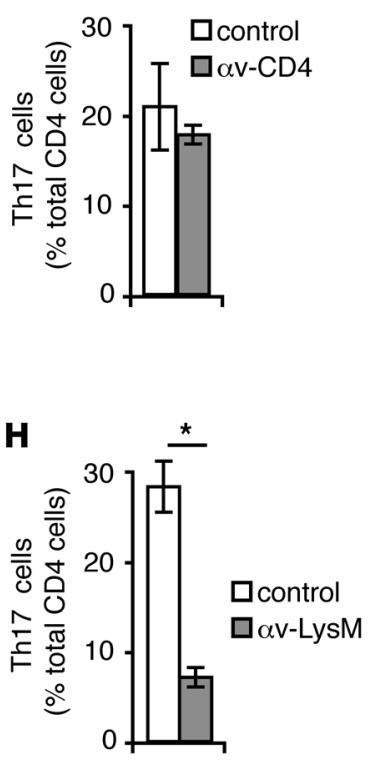
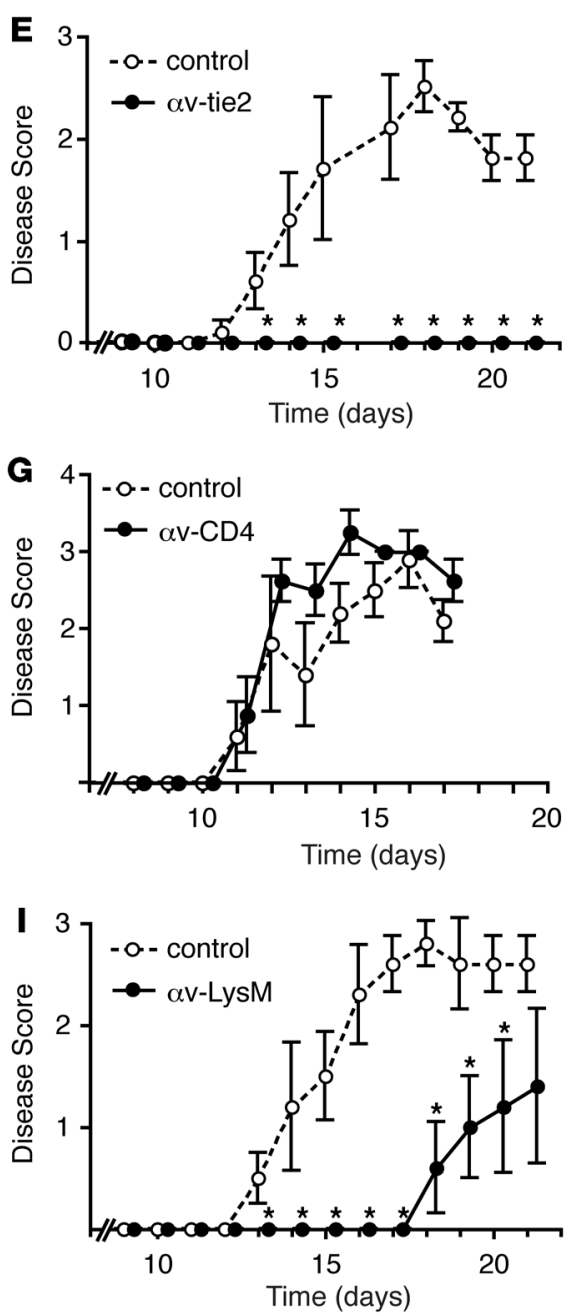

Figure 3

$\alpha V$-tie2 mice are protected from EAE. (A) Percentage of $\mathrm{CD}^{+}{ }^{+} \mathrm{T}$ cells that express IL-17 in spleen and LNs of $\alpha v$-tie 2 and control mice before immunization (day 0 ) and 10 days after immunization with MOG peptide in CFA (+10). (B-D) Percentage of CD4+ cells (B) or IL-17- and IFN- $\gamma$-producing cells ( $\mathbf{C}$ and $\mathbf{D})$ in leukocytes isolated from brain 21 days after immunization. (C) representative FACS data gated on $\mathrm{CD}^{+}$cells. (D) Percentage of $\mathrm{CD}^{+}$cells that expressed IL-17. (E) Progression of EAE in control and $\alpha v$-tie2 mice. Similar data were seen in 3 independent experiments. ( $\mathbf{F}$ and H) IL-17-producing $T$ cells in the brain of $\alpha \mathrm{V}$-CD4 mice (F) and $\alpha \mathrm{V}$-LysM mice (H) 21 days after induction of EAE. (G and I) Development of EAE in control and $\alpha v$-CD4 mice (G) or $\alpha v$-LysM mice (I). Similar results were seen in 3 ( $\alpha$ v-tie2 data) or 2 ( $\alpha$ v-LysM) independent experiments. For all graphs, data are presented as mean \pm SEM from at least 4 mice/group. ${ }^{*} P<0.05$, Student's $t$ test. prevent Th17 differentiation. cRGD had no effect on Th17 cell differentiation in the presence of active TGF- $\beta$ in both wild-type and $\alpha v$-deficient DCs. In contrast, wild-type DCs treated with cRGD no longer responded to latent TGF- $\beta$, reducing Th17 generation to the levels seen when $\alpha v$-deficient DCs were used (Figure $4 F)$. cRGD had no effect on co-cultures of T cells and $\alpha v$-deficient DCs, confirming that the CRGD was mediating its effects by inhibition of $\alpha v$ integrins on DCs. These results support a role for DC $\alpha v$ in TGF- $\beta$ activation. Moreover, they demonstrate that the activation of TGF- $\beta$ by DCs could be manipulated pharmacologically by the addition of CRGD peptides.
To establish whether this pharmacological approach could be used therapeutically, we tested whether cRGD could block development of Th17-mediated pathology in vivo. Wild-type mice treated with $\mathrm{CRGD}$ for 1 week following immunization with MOG peptide generated fewer Th17 cells than mice treated with an inactive control peptide (Figure 5, A and B). Furthermore, cRGD-treated mice were protected from EAE; over half of the mice $(3 / 5)$ developed no major signs of EAE, and the remaining mice developed disease that started later and was significantly less severe than in control mice (Figure $5 \mathrm{C})$. Hence, $\mathrm{cRGD}$ administered during $\mathrm{T}$ cell differentiation 

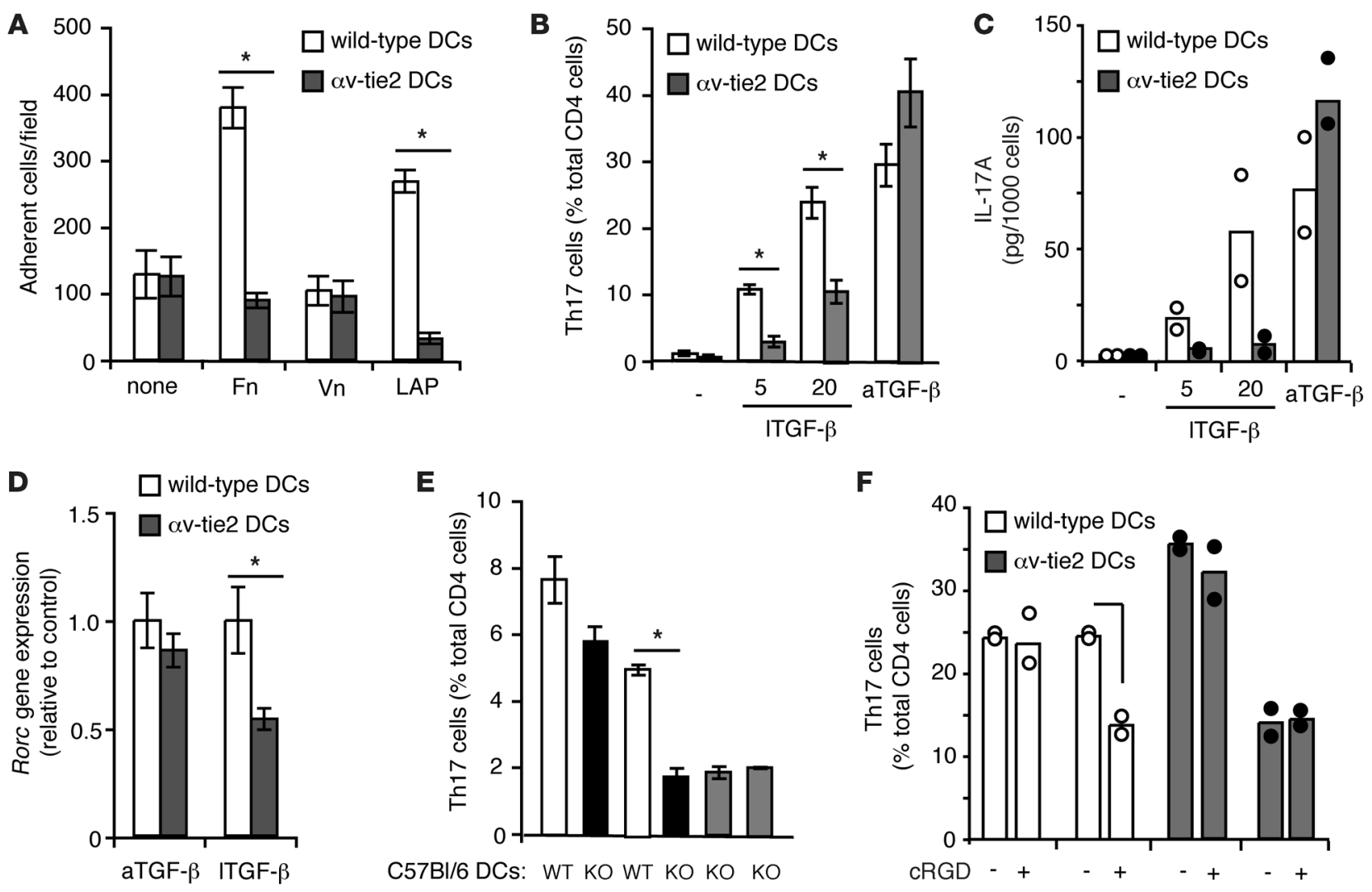

Figure 4

Expression of $\alpha$ v integrins on DCs is required for T cell responses to latent TGF- $\beta$. (A) DCs bound LAP though $\alpha v$ integrins. The number of control and $\alpha \mathrm{V}$-knockout DCs binding to untreated plates or plates coated with LAP, fibronectin (Fn), or vitronectin (Vn) is shown. Data are mean \pm SD of 3 wells. (B) Proportion of IL-17-producing T cells generated after in vitro culture of control T cells with DCs from control and $\alpha \mathrm{V}$-tie2 mouse spleens in the presence of anti-CD3, IL-6, and active TGF- $\beta$ (aTGF- $\beta$ ) or latent TGF- $\beta$ (ITGF- $\beta$ ) (5 or $20 \mathrm{ng} / \mathrm{ml})$. Data are from 3 separate DC preparations; histograms show the mean \pm SEM. Similar results were seen in at least 5 independent experiments. (C and D) Production of IL-17A protein in culture supernatant (C) and mRNA expression of Rorc (D) in T cells stimulated as in C. (E) Contact with DCs was required for T cells to respond to TGF- $\beta$ activated by $\alpha$. Proportion of IL-17-producing T cells generated after culture of T cells from OT2 TCR transgenic mice incubated with the indicated combinations of DCs from C57BL/6 and BALB/c background mice in the presence of OVA peptide, IL-6, and active or latent TGF- $\beta$. (F) cRGD peptides inhibited TGF- $\beta$ activation by DCs. Experimental conditions were the same as in B, with T cells cultured with DCs in the presence of anti-CD3, IL-6, aTGF- $\beta$, ITGF- $\beta$, and cRGD peptides. Dots represent DC cultures from independent control or $\alpha$ V-tie2 mice. Data are from 2 separate DC preparations from either control or $\alpha v$-tie2 mice. Similar results were seen in 3 independent experiments.

blocked Th17 differentiation and prevented later development of disease, consistent with inhibition of TGF- $\beta$ activation by cRGD during initiation of Th17 responses.

\section{Discussion}

In summary, our data demonstrate a critical role for DC $\alpha$ v integrins in generating Th17 cells in normal homeostatic immunity or in pathological settings. We propose that this is due to the failure of $\alpha v$-deficient DCs to activate latent TGF- $\beta$, which is required for $\mathrm{T}$ cell differentiation into Th17 cells and aTregs. Consequently, mice in which $\alpha v$ is deleted from myeloid cells did not develop Th17 cells in the intestine or in the periphery following immunization and were protected from Th17-mediated inflammatory disease. Furthermore, pharmacological inhibition of $\alpha v$ integrins impaired Th17 differentiation in vivo and ameliorated Th17-mediated pathology in a mouse model.
These studies complement and build on the work of others (23) and on our previous work (21), in which we demonstrated that $\alpha \mathrm{v}$ integrins were required for generation of aTregs in the intestine. We previously postulated that $\alpha \mathrm{v}$ integrins on myeloid cells are essential for $\mathrm{T}$ cell responses to TGF- $\beta$ by facilitating activation of latent TGF- $\beta$. Here we provide direct in vitro evidence for this hypothesis and demonstrate that this mechanism underlies DC control of generation of both Tregs and Th17 cells. Th17 cells and aTregs appear to share a common precursor and a requirement for TGF- $\beta$ signaling during early development, and the loss of both populations of cells in $\alpha v$-deficient mice is consistent with this. However, our data show that $\alpha v$ deletion causes a greater reduction in Th17 cells than in FoxP3 $3^{+}$Tregs (Figures 1 and 2), which are only depleted in the intestine and are actually found at higher numbers in mesenteric LNs (MLNs) of $\alpha v$-tie2 mice (21). This probably reflects the existence of a separate population of FoxP3 $3^{+}$Tregs (innate or natural Tregs) 


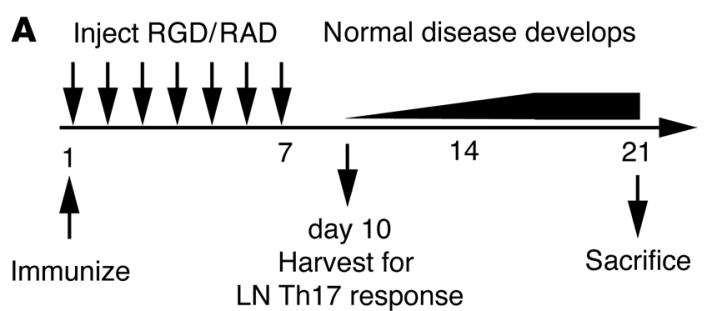

B
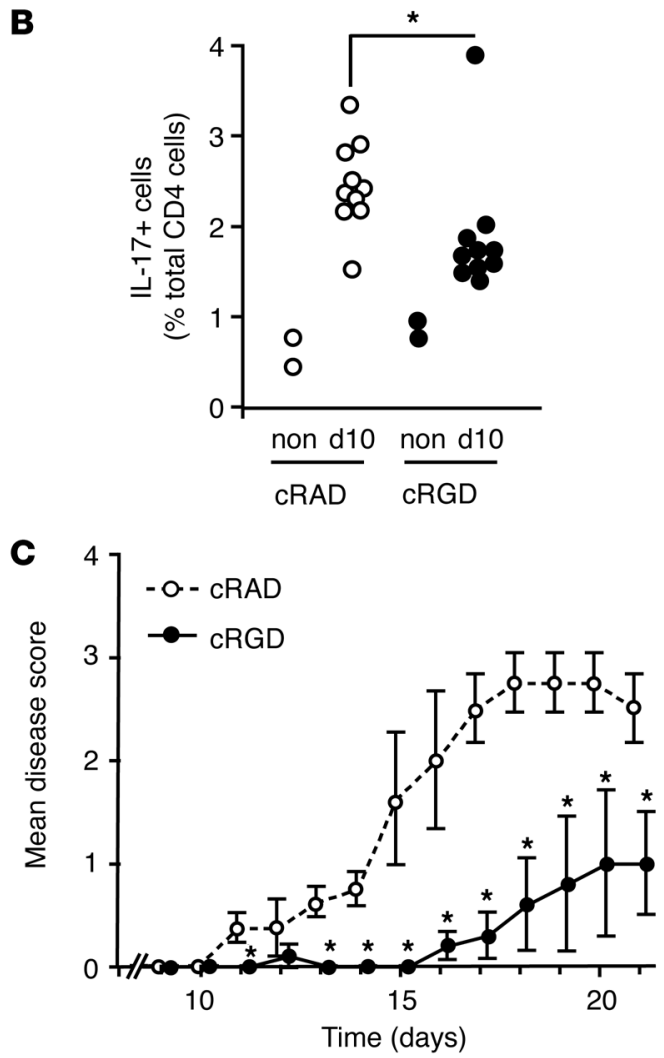

that develop independently of TGF- $\beta$ signaling (27). However, it is also possible that aTregs are less dependent on DC-mediated TGF- $\beta$ activation than Th17 cells due to greater sensitivity to TGF- $\beta$, as was proposed by Littman and colleagues (17), or to alternative mechanisms of TGF- $\beta$ activation.

Th17 cells are increasingly implicated as pathogenic effectors in inflammatory disorders in humans, including rheumatoid arthritis, psoriasis, and multiple sclerosis, and our data support such a role, as $\alpha v$-tie 2 mice lacking Th17 cells are protected in an auto-inflammatory disease model, EAE. Th17 cells have also been implicated in inflammatory bowel disease $(28,29)$, and high levels of IL-17A and other Th17-derived cytokines are found in inflamed mucosa of patients with either Crohn disease or ulcerative colitis (30). Genome-wide association studies have also highlighted Th17-conditioning cytokine IL-23 and other genes in the Th17 pathway as potential contributors to inflammatory bowel disease $(12,31-34)$. However, $\alpha v$-tie 2 mice develop spontaneous colitis, as we described previously (21), and our data therefore show that Th17 cells are not required for intestinal inflammation in mice. Furthermore, a number of recent studies in mouse models suggest that Th17 cells may actually be protective against colitis and

\section{Figure 5}

$\alpha v$ Blockade inhibits Th17 cell differentiation and protects from EAE in vivo. (A) Schematic of experiments to assess effects of cRGD on EAE progression. Mice were immunized (day 1) and given daily injections of cRGD or control peptide for 7 days, and their immune response was assessed at day 10, with paralysis monitored until day 21. (B) Percentage of LN CD4+ $\mathrm{T}$ cells that expressed IL-17 ten days after immunization (d10), or non-immunized mice (non) that also received cRAD/cRGD for 7 days. Each point represents an individual mouse, and data are combined from 2 independent experiments. ${ }^{*} P<0.05$, Student's $t$ test ( $n=9 \mathrm{cRAD}, n=10 \mathrm{cRGD}$ ). (C) Progression of EAE in cRGD- and cRAD-treated mice. Data are mean \pm SEM from at least 5 mice/group. ${ }^{*} P<0.05$, Student's $t$ test.

act to maintain epithelial integrity during infection and immunity $(7,8,35)$. It is possible, therefore, that the loss of Th17 cells in av-deficient mice contributes to the development of spontaneous colitis, which we had previously attributed principally to the loss of aTregs. In this regard, it is important to recognize that despite the lack of Th17 cells in $\alpha v$-tie 2 mice, other lymphocyte sources of IL-17 were largely unaffected by $\alpha$ v deletion, and IL-17A expression in the colon was not significantly changed. Hence, associations of inflammatory bowel disease with increased IL-17 do not necessarily implicate Th17 cells, and further studies with mice such as $\alpha v$-tie2, which lack Th17 cells rather than Th17-derived cytokines, will be important to understand the function of this complex $\mathrm{T}$ cell subset in disease.

In this study, we have identified $\alpha v$ integrin as a new component of the mechanism by which DCs generate Th17 cells. Although we have not directly addressed the identity of the $\beta$ subunit that is required for generation of Th17 cells, conditional deletion of $\beta 8$ in DCs similarly is associated with colitis and loss of intestinal Tregs (23), strongly implicating $\alpha v \beta 8$ in activation of TGF- $\beta$ and associated Th17 cell generation by DCs. Our data further suggest that pharmacological disruption of this DC/ $\alpha v \beta 8 /$ TGF- $\beta$ dialogue is a potential therapeutic option in Th17-mediated diseases.

\section{Methods}

Mice. $\alpha v$-tie2 and $\alpha v$-LysM mouse strains (21) were both backcrossed to $\mathrm{C} 57 \mathrm{BL} / 6$ mice for at least 9 generations. $\alpha v-\mathrm{CD} 4$ mice were generated by crossing C57BL/ 6 background (9 generations) $\alpha v$-knockout and $\alpha v$-floxed mice with C57BL/6 CD4 Cre transgenic mice (36). In all cases, experimental mice carried 1 floxed and 1 knockout allele of Itgav with the respective CRE transgene; littermate mice with 1 floxed, 1 wild-type Itgav allele, and the CRE transgene were used as controls. For adoptive transfer experiments, $\alpha v$-tie 2 mice backcrossed 10 generations to BALB/c background were crossed into BALB/c SCID mice to generate $\alpha v$-tie2; SCID mice and control SCID mice. $\mathrm{CD} 4^{+} \mathrm{T}$ cells were purified from spleens of control 6- to 10-week-old BALB/c mice by MACS, and $3 \times 10^{6} \mathrm{CD} 4^{+}$cells were injected intravenously into 6-week-old $\alpha \mathrm{v}$-tie2 SCID and littermate control mice. Mice were sacrificed 6 weeks later and intestines analyzed for $T$ cell development. All animals were housed under specific pathogen-free conditions at Massachusetts General Hospital and Massachusetts Institute of Technology. Animal experiments were approved by the Subcommittee on Research Animal Care at Massachusetts General Hospital or the Division of Comparative Medicine at Massachusetts Institute of Technology.

Lymphocyte isolation. Lymphocytes were harvested from lymphoid tissue by physical disruption and filtering through $40-\mu \mathrm{m}$ strainer in PBS $/ 2 \%$ BSA/2 mM EDTA (MACS buffer). Splenocytes were depleted of $\mathrm{rbc}$ ( $\mathrm{rbc}$ lysis buffer; Sigma-Aldrich). For isolation of LP lymphocytes, small intes- 
tine and colon were dissected from fat and Peyer's patches and washed to remove fecal content. Tissue was shaken three times for 15 minutes each time in $\mathrm{HBSS} / 2 \mathrm{mM}$ EDTA at $37^{\circ} \mathrm{C}$ to remove intraepithelial lymphocytes, digested in HBSS containing DNaseI $(20 \mathrm{mg} / \mathrm{ml})$ and Liberase $(0.33 \mathrm{mg} / \mathrm{ml}$; both from Roche) for 30 minutes, and incubated in PBS/2\% BSA $/ 10 \mathrm{mM}$ EDTA. Leukocytes were purified using a $40 \% / 80 \%$ percoll gradient (GE Healthcare), washed, and resuspended in MACS buffer or T cell medium for experiments.

$E A E$. For induction of EAE, mice of more than 12 weeks age were immunized subcutaneously with $200 \mu \mathrm{g} \mathrm{MOG}_{35-55}$ peptide (Hooke Laboratories) emulsified in CFA (containing $400 \mu \mathrm{g}$ Mycobacterium tuberculosis $\mathrm{H} 37 \mathrm{Ra}$ ). Mice also received $250 \mathrm{ng}$ of pertussis toxin (Hooke Laboratories) intraperitoneally at the time of immunization and 1 day later. For RGD blockade of $\alpha$ v integrins, $50 \mu \mathrm{g}$ cRGD peptide in PBS (Enzo Life Sciences) was injected subcutaneously daily from immunization until day 7 . The control group received $50 \mu \mathrm{g}$ of $\mathrm{cRAD}$ peptide instead. Mice were monitored daily for clinical signs of paralysis and scored as follows: 0 , no disease; 1 , limp tail; 2 , weak/partially paralyzed hind legs; 3 , limp tail and complete paralysis of hind legs; 4, complete hind and partial front leg paralysis; 5, complete paralysis/death. Female mice were used for EAE studies, except in experiments with $\alpha \mathrm{v}$-CD4 CRE, in which predominantly male mice were used, which resulted in a slightly accelerated and more variable disease than with females.

Isolation of brain-infiltrating cells. Brains from mice with EAE were collected in ice-cold $\mathrm{HBSS} / 10 \%$ fetal bovine serum, disrupted through a $100-\mu \mathrm{m}$ strainer, and washed, and leukocytes were purified using a 37\%/70\% percoll gradient. Mononuclear cells collected from the 37\%/70\% interface were washed with HBSS/serum and used immediately for experiments. Cells from individual brains were processed separately.

In vitro $T$ cell differentiation. Naive $\mathrm{CD} 4^{+} \mathrm{CD} 25^{-} \mathrm{T}$ cells were sorted by magnetic separation using $\mathrm{CD} 4{ }^{+} \mathrm{CD} 25^{+}$regulatory $\mathrm{T}$ cell selection kit (Stem Cell Technologies). Purity of more than $95 \%$ was routinely achieved. $\mathrm{T}$ cells were cultured in X-VIVO15 medium (Lonza) supplemented with $100 \mathrm{U} / \mathrm{ml}$ penicillin, $100 \mu \mathrm{g} / \mathrm{ml}$ streptomycin, $50 \mu \mathrm{M} 2-\beta$-mercaptoethanol for all experiments except RGD blockade, which was carried out in RPMI 1640 supplemented with $10 \%$ fetal bovine serum, $2 \mathrm{mM} \mathrm{L}$-glutamine, $100 \mathrm{U} / \mathrm{ml}$ penicillin, $100 \mu \mathrm{g} / \mathrm{ml}$ streptomycin, and $50 \mu \mathrm{M} 2-\beta$-mercaptoethanol. Splenic DCs were sorted to more than $95 \%$ purity using CD $11 \mathrm{c}$ microbeads (Miltenyi Biotech). Cells were cultured in $2 \mathrm{ml}$ volume containing $1 \times 10^{5}$ DCs and $2.5 \times 10^{5}$ naive $\mathrm{CD}^{+} \mathrm{T}$ cells in the presence of $0.5 \mu \mathrm{g} / \mathrm{ml}$ anti-CD3 (145-2C11; BD Biosciences). Additional cytokines used were recombinant active TGF- $\beta 1(1 \mathrm{ng} / \mathrm{ml})$, purified human latent TGF- $\beta(20 \mathrm{ng} / \mathrm{ml})$, and IL-6 $\left(20 \mathrm{ng} / \mathrm{ml}\right.$ ) (all R\&D Systems). For antigen-specific T cell stimulation, $1 \times 10^{5}$ DCs from mice on a C57BL/ 6 background were incubated with $2.5 \times 10^{5}$ naive $\mathrm{CD}^{+} \mathrm{T}$ cells from OT-2 TCR transgenic mice, with $100 \mathrm{nM}$ $\mathrm{OVA}_{323-339}$ peptide and cytokines, in the presence or absence of $1 \times 10^{5}$ DCs from BALB/c-background mice. For RGD blockade experiments, cRGD peptide was added at $2 \mu \mathrm{g} / \mathrm{ml}$. Cells were cultured for 4 or 5 days before analysis of Th17 differentiation. IL-17A production was measured by intracellular cytokine staining, as described below, or by IL-17A release

into the culture supernatant, measured using cytokine bead array (BD Biosciences). Expression of the Th17-specific transcription factor was measured by quantitative real-time PCR (QRT-PCR), as described below.

Flow cytometry and intracellular cytokine staining. For intracellular cytokine staining, cells obtained from dissection of LP, mesenteric and peripheral LNs, Peyer's patches, spleens, in vitro cultures, or brains were resuspended in T cell medium and stimulated for 5 hours with $10 \mathrm{ng} / \mathrm{ml} \mathrm{PMA} / 750 \mathrm{ng} / \mathrm{ml}$ ionomycin in the presence of intracellular transport inhibitors $(1 \mu \mathrm{g} / \mathrm{ml}$ GolgiPlug; BD Biosciences). Cells were treated with Fc-blocking mAbs and surface stained with anti-CD4-APC (RM4-5), anti-CD8 $\alpha$-PerCP (53-6.7), or anti-TCR- $\gamma-\mathrm{APC}$ (GL3), followed by intracellular cytokine staining with anti-IL-17-PE (TC11-18H10), anti-IFN- $\gamma$-FITC (XMG1.2), anti-ROR $\gamma$-PE (AFKJS-9), and anti-Foxp3-FITC (FJK-16s) according to the manufacturer's instructions (BD Biosciences or eBioscience). Data were acquired using FACSCalibur instrument (BD Biosciences) and analyzed using FlowJo software (Tree Star Inc.). Percentage and absolute numbers of cytokine-expressing cells within the $\mathrm{CD}^{+}, \mathrm{CD}^{+}$, or TCR- $\gamma^{+}$populations were determined.

QRT-PCR. RNA was prepared from $0.5 \mathrm{~cm}$ of the proximal colon of $\alpha \mathrm{v}$-tie2 mice and littermate controls of 6-8 weeks of age or from cultured $T$ cells at day 4 of culture in Th17-generating conditions using Trizol (Invitrogen). cDNA was synthesized using reverse transcription reagent (Applied Biosystems). cDNA was analyzed by Fast start SYBR Green reagents (Roche) using a RealPlex Mastercycler (Eppendorf) and the inflammatory cytokine and receptors rtPCR array (SA Biosciences) or the following primers: $I l 17 a$ 5'-GAAGATGCTGGTGGGTGTGG-3' and 5'AGCCGCGGGTCTCTGTTTAG-3'; Ill17f 5'-TGAATTCCAGAACGCTCCA-3' and 5'-TCTTCCTGACCCTGGGCATT-3'; Rorc 5'-CCGCTGAGAGGGCTTCAC-3' and 5'-TGCAGGAGTAGGCCACATTACA-3'. Expression levels of each gene were normalized to $\beta$-actin expression.

\section{Acknowledgments}

This work was supported by grants from the Hood Pediatric Research Foundation (to A. Lacy-Hulbert) and the Howard Hughes Medical Institute (R.O. Hynes) and by a Wellcome Trust Travelling Fellowship (to S. Mukhopadhyay). Helena Païdassi was supported by MRC grant G0802069 to John Savill, University of Edinburgh, who we thank for support and helpful discussion.

Received for publication May 24, 2010, and accepted in revised form September 29, 2010.

Address correspondence to: Adam Lacy-Hulbert, Program of Developmental Immunology, Massachusetts General Hospital GRJ1402, 55 Fruit Street, Boston, Massachusetts 02114, USA. Phone: 617.643.5346; Fax: 617.724.3248; E-mail: alacy-hulbert@ partners.org.

Stephan Kissler's present address is: Rudolf Virchow Center, DFG Center for Experimental Biomedicine, University of Würzburg, Würzburg, Germany.

1. Harrington LE, et al. Interleukin 17-producing CD4+ effector T cells develop via a lineage distinct from the T helper type 1 and 2 lineages. Nat Immunol. 2005;6(11):1123-1132.

2. Park H, et al. A distinct lineage of CD4 T cells regulates tissue inflammation by producing interleukin 17. Nat Immunol. 2005;6(11):1133-1141.

3. Korn T, Bettelli E, Oukka M, Kuchroo VK. IL-17 and Th17 cells. Annu Rev Immunol. 2009;27:485-517.

4. Steinman L. A brief history of $\mathrm{T}(\mathrm{H}) 17$, the first major revision in the $\mathrm{T}(\mathrm{H}) 1 / \mathrm{T}(\mathrm{H}) 2$ hypothesis of T cell-mediated tissue damage. Nat Med. 2007;
13(2):139-145.

5. Ivanov II, et al. The orphan nuclear receptor RORgammat directs the differentiation program of proinflammatory IL-17+ T helper cells. Cell. 2006;126(6):1121-1133.

6. Ivanov II, et al. Specific microbiota direct the differentiation of IL-17-producing T-helper cells in the mucosa of the small intestine. Cell Host Microbe. 2008;4(4):337-349.

7. Ivanov II, et al. Induction of intestinal Th17 cells by segmented filamentous bacteria. Cell. 2009;139(3):485-498.
8. Zenewicz LA, Yancopoulos GD, Valenzuela DM, Murphy AJ, Stevens S, Flavell RA. Innate and adaptive interleukin-22 protects mice from inflammatory bowel disease. Immunity. 2008; 29(6):947-957.

9. O'Connor W Jr, et al. A protective function for interleukin 17A in $\mathrm{T}$ cell-mediated intestinal inflammation. Nat Immunol. 2009;10(6):603-609.

10. Cua DJ, et al. Interleukin-23 rather than interleukin12 is the critical cytokine for autoimmune inflammation of the brain. Nature. 2003;421(6924):744-748.

11. Lock C, et al. Gene-microarray analysis of mul- 
tiple sclerosis lesions yields new targets validated in autoimmune encephalomyelitis. Nat Med. 2002;8(5):500-508.

12. Duerr RH, et al. A genome-wide association study identifies IL23R as an inflammatory bowel disease gene. Science. 2006;314(5804):1461-1463.

13. Lee YK, et al. Late developmental plasticity in the T helper 17 lineage. Immunity. 2009;30(1):92-107.

14. Veldhoen M, Hocking RJ, Atkins CJ, Locksley RM, Stockinger B. TGFbeta in the context of an inflammatory cytokine milieu supports de novo differentiation of IL-17-producing T cells. Immunity. 2006; 24(2):179-189

15. Mangan PR, et al. Transforming growth factor-beta induces development of the $\mathrm{T}(\mathrm{H}) 17$ lineage. Nature. 2006;441(7090):231-234

16. Bettelli E, et al. Reciprocal developmental pathways for the generation of pathogenic effector TH17 and regulatory T cells. Nature. 2006;441(7090):235-238.

17. Zhou L, et al. TGF-beta-induced Foxp3 inhibits $\mathrm{T}(\mathrm{H}) 17$ cell differentiation by antagonizing RORgammat function. Nature. 2008;453(7192):236-240.

18. Munger JS, et al. The integrin alpha $v$ beta 6 binds and activates latent TGF beta 1: a mechanism for regulating pulmonary inflammation and fibrosis. Cell. 1999;96(3):319-328.

19. Aluwihare $P$, et al. Mice that lack activity of $\{$ alpha $\}$ $v\{$ beta $\} 6-$ and $\{$ alpha $\}$ v $\{$ beta $\} 8$-integrins reproduce the abnormalities of Tgfb1- and Tgfb3-null mice.
J Cell Sci. 2009;122(pt 2):227-232.

20. Yang $Z$, et al. Absence of integrin-mediated TGFbeta 1 activation in vivo recapitulates the phenotype of TGFbeta1-null mice. J Cell Biol. 2007;176(6):787-793.

21. Lacy-Hulbert A, et al. Ulcerative colitis and autoimmunity induced by loss of myeloid alpha (v) integrins. Proc Natl Acad Sci U S A. 2007;104(40):15823-15828.

22. Das J, et al. Transforming growth factor beta is dispensable for the molecular orchestration of Th17 cell differentiation. J Exp Med. 2009;206(11):2407-2416.

23. Travis MA, et al. Loss of integrin alpha(v)beta(8) on dendritic cells causes autoimmunity and colitis in mice. Nature. 2007;449(7160):361-365

24. Munger JS, Harpel JG, Giancotti FG, Rifkin DB. Interactions between growth factors and integrins: latent forms of transforming growth factor-beta are ligands for the integrin alphavbeta1. Mol Biol Cell. 1998;9(9):2627-2638.

25. Reardon DA, Nabors LB, Stupp R, Mikkelsen T. Cilengitide: an integrin-targeting arginine-glycineaspartic acid peptide with promising activity for glioblastoma multiforme. Expert Opin Investig Drugs. 2008;17(8):1225-1235.

26. Kumagai H, Tajima M, Ueno Y, Giga-Hama Y, Ohba M. Effect of cyclic RGD peptide on cell adhesion and tumor metastasis. Biochem Biophys Res Commun. 1991; 177(1):74-82.

27. Bluestone JA, Abbas AK. Natural versus adaptive regulatory T cells. Nat Rev Immunol. 2003;3(3):253-257.
28. Liu ZJ, Yadav PK, Su JL, Wang JS, Fei K. Potential role of Th17 cells in the pathogenesis of inflammatory bowel disease. World J Gastroenterol. 2009; 15(46):5784-5788.

29. Abraham C, Cho J. Interleukin-23/Th17 pathways and inflammatory bowel disease. Inflamm Bowel Dis. 2009;15(7):1090-1100.

30. Fujino S, et al. Increased expression of interleukin 17 in inflammatory bowel disease. Gut. 2003; 52(1):65-70.

31. Barrett JC, et al. Genome-wide association defines more than 30 distinct susceptibility loci for Crohn's disease. Nat Genet. 2008;40(8):955-962.

32. Fisher SA, et al. Genetic determinants of ulcerative colitis include the ECM1 locus and five loci implicated in Crohn's disease. Nat Genet. 2008;40(6):710-712.

33. Franke A, et al. Sequence variants in IL10, ARPC2 and multiple other loci contribute to ulcerative colitis susceptibility. Nat Genet. 2008;40(11):1319-1323.

34. Franke A, et al. Replication of signals from recent studies of Crohn's disease identifies previously unknown disease loci for ulcerative colitis. Nat Genet. 2008;40(6):713-715.

35. Ahern PP, Izcue A, Maloy KJ, Powrie F. The interleukin-23 axis in intestinal inflammation. Immunol Rev. 2008;226:147-159.

36. Lee PP, et al. A critical role for Dnmt1 and DNA methylation in T cell development, function, and survival. Immunity. 2001;15(5):763-774. 